\title{
GPR18 Agonist Resolvin D2 Reduces the Early Brain Injury in A Rat Model of Subarachnoid Hemorrhage by Multi-Mechanisms of Protection
}

Tongyu Zhang ( $\boldsymbol{\sim}$ dr_tongyuzhang@ccmu.edu.cn )

Xuanwu Hospital Department of Neurosurgery https://orcid.org/0000-0003-1383-8278

Gang Zuo

The Affiliated Taicang Hospital, Soochow University

Hongqi Zhang

Xuanwu Hospital Department of Neurosurgery

\section{Research Article}

Keywords: GPR18, Resolvin D2, The whole brain, Early brain injury, Subarachnoid hemorrhage

Posted Date: April 8th, 2021

DOI: https://doi.org/10.21203/rs.3.rs-398477/v1

License: (c) (i) This work is licensed under a Creative Commons Attribution 4.0 International License.

Read Full License 


\section{Abstract}

Background Early brain injury (EBI) is the early phase of secondary complications resulted in poor prognosis of subarachnoid hemorrhage (SAH). GPR18 is a G protein-coupled receptor which has been reported for neuroprotection in ischemia. In this study, we aimed to use resolvin D2 (RvD2) as an agonist to investigate the roles of GPR18 in different brain regions during EBI. Methods Location and time course of GPR18 after SAH were measured with immunofluorescence and western blot in endovascular perforation rat model. RvD2 was given one hour intranasally post-SAH, and SAH grades, neurobehavior and brain water content tests were performed after 24 hours. TUNEL and DHE staining were measured 24 hours post-SAH in cortex. Immunofluorescence, western blot and immunohistochemistry of proteins related to EBI in different brain regions were also performed. Results We found GPR18 mainly located in meninges, hypothalamus, cortex and white matter. And GPR18 expression increased in meninges and hypothalamus after EBI, however, it decreased in cortex and white matter. RvD2 could improve neurological scores and brain edema. Mast cell degranulation was attenuated, Chymase and Typtase expression decreased after RvD2 administration in meninges. RvD2 attenuated inflammation with increase of POMC, IL-10 and decrease of NPY, TNF- $a$ in hypothalamus. In cortex, RvD2 alleviated oxidative stress and apoptosis, protected blood-brain barrier. RvD2 also ameliorated white matter injury by MBP elevation and APP depression. Conclusions Current results emphasized the importance of GPR18 in the whole brain during EBI. Upregulation of GPR18 with RvD2 may improve neurological functions with multi-mechanisms in different brain regions.

\section{Introduction}

Subarachnoid hemorrhage (SAH) is a cerebrovascular emergency associated with high morbidity and mortality (Macdonald and Schweizer 2017). Early brain injury (EBI) within $72 \mathrm{~h}$ after SAH is the key factor leading to acute neurological dysfunction in SAH patients (Chen et al. 2014; Topkoru et al. 2017). In fact, $30 \%$ of SAH patients die within 72 hours after the onset of EBI (Topkoru et al. 2017). Therefore, reducing the occurrence of EBI and early intervention in SAH may help improve prognosis (Chen et al. 2014). One challenge to achieving these goals is that EBI affects the entire brain (Pang et al. 2018) through various mechanisms such as inflammation, blood-brain barrier (BBB) disruption, apoptosis, oxidative stress, white matter injury (WMI) (Chen et al. 2017). Pro-inflammatory reactions and free radicals at acute stage of SAH may contribute to cell death and cerebral vasospasm, whereas they might be required for neurovascular remodeling and neurogenesis in later stage (Ciurea et al. 2013).

Resolvin D2 (RvD2), derived from omega-3 fatty acids and a precursor of docosahexaenoic acid (DHA), can strongly inhibit leukocytes (Siddiqui et al. 2019). RvD2 is also able to cross the BBB effectively through its specific receptor GPR18, and it exerts anti-inflammatory effects in various neurological diseases (Dong et al. 2019; Zuo et al. 2018). Low-dose RvD2 reduces inflammatory pain induced by formalin (Zhang et al. 2018a). Our previous study revealed that RvD2 could protect neuronal and endothelial cells from cerebral ischemia/reperfusion injury (Zuo et al. 2018). However, whether RvD2 can protect the brain from SAH injury is unclear. 
RvD2 is a selective ligand of G Protein-coupled Receptor 18 (GPR18), a G protein-coupled receptor (GPCR) expressed in a wide range of tissues, including brain (Dunn et al. 2016). RvD2 and the $\mathrm{G}_{\text {as }}$ subunit of GPR18 may interact to improve phagocyte functions and promote organ protection (Chiang et al. 2015). Elevated GPR18 expression, especially in hypothalamus, was shown to down-regulate the immune response in inflammatory disorders (Pascoal et al. 2017). GPR18 activation could also decrease neuronal apoptosis and integrity of the BBB in the cortex (Zuo et al. 2018). To what extent RvD2 does, or could, contribute to these potentially protective effects of GPR18 is unclear. Since we previously reported that DHA can improve neurological function after SAH (Zhang et al. 2019a), and since RvD2 is a precursor of DHA, we wondered whether RvD2 might also exert protective effects in SAH. Therefore, the present study aimed to examine the potential role of RvD2 on the GPR18 axis in different brain regions during SAHinduced EBI.

\section{Material And Methods}

\section{Animals}

A total of 135 adult male Sprague-Dawley rats (220-320 g, 6-8 weeks old) were purchased from Beijing Vital River Laboratory Animal Technology and housed in the laboratory animal center of Xuanwu Hospital at Capital Medical University (Beijing, China). Animals were maintained in facilities at constant temperature $\left(25^{\circ} \mathrm{C}\right)$ with humidity control on a 12/12-h light/dark cycle, with free access to food and water. All procedures were approved by the Institutional Animal Care and Use Committee of the Xuanwu Hospital in accordance with the guidelines of the US National Institutes of Health "Guide for the Care and Use of Laboratory Animals".

\section{Experimental SAH model}

A rat model of SAH was established using endovascular perforation as described (Zhang et al. 2018b). In brief, rats were anesthetized with $3 \%$ isoflurane in $35 \%$ medical oxygen, delivered through tracheal intubation and a rodent ventilator (Harvard Apparatus, Holliston, MA, USA). Then a sharpened 4-0 nylon suture (DOTMED, New York City, NY, USA) was placed in the left external carotid artery and punched through the left internal carotid artery until resistance was felt. The suture further punctured the vessel for 3 seconds, after which it was withdrawn. Sham-operated rats underwent the same procedures without perforation with the suture.

\section{Experimental design}

The rats were randomly distributed into the following groups: Naïve $(n=3)$, Sham $(n=9)$, Sham + vehicle $(n=18), S A H(n=33), S A H+$ vehicle $(n=18), S A H+0.3 \mu g / k g ~ R v D 2(n=6), S A H+0.9 \mu g / k g ~ R v D 2(n=$ $18)$, and $\mathrm{SAH}+2.7 \mu \mathrm{g} / \mathrm{kg} \operatorname{RvD} 2(\mathrm{n}=6)$. The groups were treated as follows and as illustrated in Fig. 1 :

Experiment 1: Location and dynamics of GPR18 expression in different brain regions after SAH 
We first measured the immunofluorescence of GPR18 in different brain regions of three naïve rats. GPR18 expression in various brain regions was also measured over time in different brain regions (Supplemental Figure 1). A total of 36 rats were randomly assigned to six groups depending on at what time point after SAH induction they were analyzed ( $n=6$ per group): Sham, SAH-3 h, SAH- 6 h, SAH- 12 h, SAH- 24 h, and $\mathrm{SAH}-72 \mathrm{~h}$. Western blot analysis was performed to assess the protein levels of GPR18. Another three Sham rats and three $\mathrm{SAH}-24 \mathrm{~h}$ rats were analyzed by double immunofluorescence staining against GPR18 as well a marker of mast cells and microglia (Supplemental Figure 2).

\section{Experiment 2: Role of RvD2 in neurological function and brain edema}

To evaluate the effects of GPR18 activation by RvD2 on neurological outcomes after $\mathrm{SAH}, 30$ rats were randomly assigned into five groups ( $n=6$ per group): Sham + vehicle, SAH + vehicle, SAH+RvD2 (0.3 $\mu \mathrm{g} / \mathrm{kg}), \mathrm{SAH}+\mathrm{RvD} 2(0.9 \mu \mathrm{g} / \mathrm{kg})$, and SAH+RvD2 $(2.7 \mu \mathrm{g} / \mathrm{kg})$. The SAH grade test, modified Garcia test, beam balance test and brain water content test were performed as described below at $24 \mathrm{~h}$ after SAH in all groups.

\section{Experiment 3: Mechanisms of RVD2-mediated GPR18 activation in different brain regions}

To explore the neuroprotective mechanisms of GPR18 activation by RvD2 in different brain regions after $\mathrm{SAH}, 36$ rats were randomly assigned to three groups ( $n=12$ per group): Sham + vehicle, $\mathrm{SAH}+$ vehicle, and SAH + RvD2 $(0.9 \mu \mathrm{g} / \mathrm{kg})$. Neurological scores were assessed before euthanasia (Supplemental Figure 3). At $24 \mathrm{~h}$ after $\mathrm{SAH}, 18$ rats ( $\mathrm{n}=6$ per group) were analyzed by processing sections from different brain regions with terminal deoxynucleotidyl transferase dUTP (TUNEL), dihydroethidium (DHE), immunofluorescence and toluidine blue. At the same time point, another 18 rats ( $n=6$ per group) were analyzed by processing lysates from different brain regions for western blotting to evaluate mast cell degranulation, hypothalamic inflammation, BBB disruption, oxidative stress, neuronal apoptosis and WMI.

\section{Administration of RvD2}

RvD2 (Sigma-Aldrich, St. Louis, MO, USA) was diluted with 1\% dimethylsulfoxide (DMSO) in sterile saline and administered intranasally at $1 \mathrm{~h}$ after induction of SAH as described above. Vehicle was $1 \%$ dimethylsulfoxide (DMSO) in sterile saline. Rats in the SAH + vehicle and Sham groups received an equal volume of vehicle with the same method of injection.

Intranasal administration was performed as our previous study reported (Zuo et al. 2019). In brief, the anesthetized rats were placed on their backs and administered with vehicle or RvD2 at a dose of $0.3,0.9$ or $2.7 \mu \mathrm{g} / \mathrm{kg}$ ) as nose drops ( $1 \mu \mathrm{l} / \mathrm{drop}$ ) over a period of $20 \mathrm{~min}$. Alternating drops were given every $2 \mathrm{~min}$ between the left and right nares. A total volume of $10 \mu \mathrm{l}$ was administered intranasally.

\section{SAH grade}


The severity of SAH was measured on the SAH grading scale by blinded investigators before euthanasia at $24 \mathrm{~h}$ after SAH surgery as previous reported (Zhang et al. 2018b). Rats with a grade lower than 8 were excluded because that previous work suggested that their injury was minimal. Thus, one animal was excluded from the SAH-3h group, two from the SAH- $6 \mathrm{~h}$ group, one from the SAH-24 h, and two from the $\mathrm{SAH}+$ vehicle group.

\section{Neurological score and brain water content}

Neurological scores were divided into the modified Garcia and beam balance scores as previously described (Zhang et al. 2018b). Six individual tests for responses to motion and sensation were performed and summarized as a modified Garcia score, while the beam balance score was expressed on a 4-point scale.

Left hemisphere, right hemisphere, cerebellum and brain stem were harvested from rats without perfusion. Each sample was weighed before drying to obtain the wet weight (WW), then dried in an oven at $105{ }^{\circ} \mathrm{C}$ to obtain the dry weight (DW). Brain water content was calculated as $[(W W-D W) / W W] \times$ $100 \%$.

\section{TUNEL staining}

TUNEL staining was carried out using the Cell Death Detection Kit (Roche, San Francisco, CA, USA) was performed as described (Zhang et al. 2019a). After anesthetization, animals were intracardially perfused with $100 \mathrm{~mL}$ ice-cold phosphate-buffered saline (PBS), followed by $100 \mathrm{~mL}$ of $10 \%$ paraformaldehyde. Whole brains were harvested and fixed in $10 \%$ paraformaldehyde for 24 hours followed by $30 \%$ sucrose for three days. After being frozen at $-80^{\circ} \mathrm{C}$, the brains were cut into sections $8 \mu \mathrm{m}$ thick on a cryostat (LM3050S; Leica Microsystems, Bannockburn, Germany). Slices were divided into Sham + vehicle, SAH + vehicle and SAH + RvD2 groups. Nuclei were stained using 4-diamidino-2-phenylindole (DAPI), and samples were analyzed under a DMI8 fluorescence microscope (Leica Microsystems, Buffalo Grove, IL, USA).

ImageJ software was used for analysis. Mean numbers of TUNEL-positive and total cells were determined for six sections per brain at ' 200 magnification by a blinded investigator. Apoptotic index was expressed as the ratio of the number of TUNEL-positive cells to the total number of cells within the temporal lobe of the cortex in each group.

\section{DHE staining}

The stock DHE reagent was purchased from Invitrogen (Grand Island, NY) and working agent for experiments was prepared as described (Zhang et al. 2019a). Briefly, brain slices were prepared as described above for TUNEL staining. After 1-h incubation with working agent and 10 min of PBS washing, the slides were dried at $37^{\circ} \mathrm{C}$ for 30 min, then mounted and washed three times with PBS, each time for 
10 min. Sections were observed and evaluated as for TUNEL staining. An oxidative stress index was calculated as the average number of DHE-positive cells per $\mathrm{mm}^{2}$ in the temporal lobe of the cortex.

\section{Toluidine blue staining}

Whole brains were harvested from rats in each group at $24 \mathrm{~h}$ after operation. Slices $8 \mu \mathrm{m}$ thick were prepared as described (Zhang et al. 2006). Each slice was dipped in toluidine blue staining solution for 30 min, gently rinsed with distilled water for 3-5 min, and developed using $0.5 \%$ glacial acetic acid until the nucleus and intracellular features were clearly visible. The sections were rapidly dehydrated in $95 \%$ ethanol, rendered transparent using xylene, then mounted, sealed and examined under a DCM8 optical microscope (Leica Microsystems, Buffalo Grove, IL, USA).

\section{Immunofluorescence staining}

Whole brains were harvested from rats in each group at $24 \mathrm{~h}$ after operation. Slices $8 \mu \mathrm{m}$ thick were prepared as described (Zhang et al. 2019b) and stained overnight at $4{ }^{\circ} \mathrm{C}$ with primary antibodies against GPR18 (1:200, Abcam, ab76258), aquaporin protein 4 (AQP4) (1:200, Abcam, ab9512), nerve peptide $Y$ (NPY) (1:100, Novus Biologicals, NBP1-46535), proopiomelanocortin (POMC) (1:200, Abcam, ab94446), chymase (1:200, Abcam, ab186417), tryptase (1:200, Abcam, ab151757), or Iba1 (1:200, Abcam, ab178846). Nuclei were stained using DAPI (Abcam, ab104139). Next, brain sections were incubated at room temperature for $2 \mathrm{~h}$ with secondary antibodies (1:500, Jackson Immunoresearch, PA, USA). Sections were observed and analyzed as described above for TUNEL staining.

\section{Western blot analysis}

Western blot analysis was performed as described (Zhang et al. 2019b). In brief, different regions of cerebral hemispheres were collected, grinded and lysed with RIPA buffer for 1 hour in room temperature, then equal amounts of total protein $(50 \mu \mathrm{g})$ were fractionated on sodium dodecylsulphate polyacrylamide gel electrophoresis (SDS-PAGE) and transferred to nitrocellulose membranes. Membranes were blocked for 2 hours with $5 \%$ non-fat milk in Tris-buffered saline containing Tween-20 (pH 7.6), followed by overnight incubation at $4{ }^{\circ} \mathrm{C}$ with primary antibody against GPR18 (1:1000, Abcam, ab76258), chymase (1:1000, Abcam, ab186417), tryptase (1:1000, Abcam, ab151757), NPY (1:1000, Novus Biologicals, NBP146535), POMC (1:1000, Abcam, ab94446), TNF-a (1:1000, Abcam, ab205587), interleukin-10 (IL-10) (1:1000, Abcam, ab133575), matrix metalloprotease-9 (MMP-9) (1:1000, Abcam, ab76003), AQP4 (1:1000, Santa Cruz Biotechnology, sc-390488), cleaved caspase-3 (1:1000, Cell Signaling Technology, \#9664), Romo1 (1:1000, Abcam, ab236409), amyloid precursor protein (APP) (1:1500, Abcam, ab101492), myelin basic protein (MBP) (1:1000, Abcam, ab209328). Membranes were immunostained against $\beta$-actin (1:5000, Santa Cruz Biotechnology, sc-8432) as a loading control. After 3 times of washing with washing buffer, The membranes were then incubated with appropriate secondary antibodies (1:5000, Santa Cruz Biotechnology). The images were analyzed in a blinded fashion using ImageJ software. 


\section{Statistical analysis}

All experiments were performed with full blinding, allocation concealment, and randomization. The Shapiro-Wilk test was used to test data for normality before analysis. Normally distributed continuous variables were expressed as mean $\pm S D$ and compared among three or more groups using one-way analysis of variance (ANOVA), followed by Tukey's post-hoc test. Skewed continuous variables were expressed as median (interquartile range) and compared between groups using the Kruskal-Wallis test, followed by Dunn's post-hoc test. $P<0.05$ was defined as statistically significant. All statistical analyses were performed using SPSS 24.0 (IBM, Chicago, IL, USA).

\section{Results}

\section{Mortality and SAH grade}

A total of 135 rats were used in this study. Fifteen rats died after the operation and six were excluded from further analysis because of low SAH grades (Fig. 2A). No animals in the Naïve, Sham or Sham + vehicle group died before euthanasia. Among the 73 animals in the SAH + vehicle group and the SAH groups analyzed at $3,6,12,24$, and $72 \mathrm{~h}$ (after excluding six animals), 16 died, giving a mortality rate of $21.92 \%$ (Fig. 2A). In contrast, only two of the 32 animals in all three SAH + RvD2 groups died, giving a mortality rate of $6.25 \%$ (Fig. 2A). Representative brain images from the Sham and SAH groups are shown in Fig. 2B. The mean SAH grade was 0 in the Naïve, Sham and Sham + vehicle groups. There was no statistical difference in SAH grade among any of the SAH groups, regardless of time point after SAH and regardless of RvD2 dose administered (Fig. 2C).

\section{Time course of GPR18 expression in different brain regions after SAH}

Immunofluorescence staining of GPR18 protein in the Naïve group showed that it expressed in meninges, hypothalamus, cortex and white matter (Fig. 3). And there was almost no expression of GPR18 in hippocampus and thalamus (Fig. 3). Temporal patterns of GPR18 were analyzed in these four brain regions after SAH (Supplemental Figure 1). In brain meninges, the expression of GPR18 increased as early as $6 \mathrm{~h}$ after SAH onset relative to the Sham group, and reached its highest level at $72 \mathrm{~h}$ (Fig. 4A, B). In brain hypothalamus, the expression of GPR18 also increased after SAH onset and peaked at $24 \mathrm{~h}$ (Fig. $4 A, C)$. In brain cortex, the expression of GPR18 decreased after SAH onset and reached the lowest level at 12 hours (Fig. 4A, D). In white matter, the expression of GPR18 decreased after SAH onset and reached the lowest level at $72 \mathrm{~h}$ (Fig. 4A, E). At $24 \mathrm{~h}$ after SAH, GPR18 expression was substantially different from the the expression in Sham animals in all four brain regions (Fig. 4A, B). These results indicate that SAH strongly affects GPR18 expression in multiple brain regions for longer periods of at least $24 \mathrm{~h}$. Thus, we chose this time point for further study.

\section{RvD2 improved neurological sores and brain edema after SAH}


To investigate the effects of RvD2 on neurological scores and brain edema after SAH, the modified Garcia score, beam balance test and brain water content were measured to assess neurological and behavioral defects. The modified Garcia and beam balance scores in the SAH groups at $24 \mathrm{~h}$ were significantly lower than those in the Sham + vehicle group (Fig. 5A). RvD2 treatment markedly improved the modified Garcia and beam balance scores compared with the SAH + vehicle group, especially at a dose of $0.9 \mu \mathrm{g} / \mathrm{kg}$ (Fig. $5 \mathrm{~A})$. There was more brain water content in the SAH groups at $24 \mathrm{~h}$ than those in the Sham + vehicle group (Fig. 5B). $0.9 \mu \mathrm{g} / \mathrm{kg}$ dose of RvD2 also reduced the brain water content percentage in the left and right hemispheres of the SAH rats (Fig. 5B). Since the medium dose of RvD2 $(0.9 \mu \mathrm{g} / \mathrm{kg})$ was able to improve the three indices, we chose it as the RvD2 concentration for all subsequent experiments. Similar neuroprotective effects of RvD2 were observed in Experiment 3 (Supplemental Figure 3).

\section{RvD2 attenuated mast cell degranulation in brain meninges after SAH}

In brain meninges, GPR18 colocalized with mast cell markers (Supplemental Figure 2A, B). Immunohistochemistry staining with toluidine blue was performed to assess mast cell degranulation. More mast cells and degranulated cells were found in the SAH + vehicle group than in the Sham + vehicle group (Fig. 6A). The degranulation of mast cells was attenuated in the SAH + RvD2 group (Fig. 6A). Meanwhile, the SAH + vehicle group showed increased expression of GPR18 and increased numbers of the mast cell degranulation markers chymase and tryptase (Fig. 6B). Administering RvD2 to SAH rats reduced the expression of chymase and tryptase but increased the expression of GPR18 (Fig. 6B).

\section{RvD2 attenuated SAH-induced inflammation in hypothalamus}

In hypothalamus, POMC and NPY are two neurons related to inflammation. Our results of immunofluorescence staining revealed that GPR18 can colocalize with NPY and POMC in neurons (Fig. $7 A)$. In the SAH + vehicle group, levels of GPR18, NPY and pro-inflammatory marker tumor necrosis factor-alpha (TNF- $a$ ) were increased, whereas the levels of POMC and anti-inflammatory marker interleukin-10 (IL-10) were decreased (Fig. 7B). Administering RvD2 to SAH rats further elevated GPR18 expression, and it reversed the SAH-induced increases in NPY, POMC, TNF-a and IL-10 (Fig. 7B).

\section{RvD2 protected the BBB and attenuated apoptosis and oxidative stress in cortex after SAH}

AQP4 levels were measured in cortex to assess BBB integrity, while TUNEL and DHE staining were carried out to assess, respectively, cellular apoptosis and oxidative stress. SAH rats showed significantly more AQP4-positive, TUNEL-positive or DHE-positive cells than Sham + vehicle (Fig. 8A-C). The SAH + RvD2 group showed fewer TUNEL-positive, AQP4-positive or DHE-positive cells than the SAH + vehicle group. At the same time, SAH rats showed a decrease in the expression of GPR18 and an increase in the expression of blood brain barrier related proteins MMP-9, AQP4, apoptosis related protein cleaved caspase-3 and oxidative stress related protein Romo1 (Fig. 9A-F). RvD2 increased the expression of GPR18 but decreased the expression of MMP-9, AQP4, cleaved caspase-3 and Romo1 (Fig. 9A-F). These results suggest that GPR18 may help to protect the BBB, attenuate apoptosis and oxidative stress in cortex following SAH. 


\section{RvD2 mitigated axonal and myelin injury in brain white matter after SAH}

In brain white matter, immunofluorescence staining and western blots showed that SAH increased the axonal injury marker APP and decreased the myelin marker MBP (Fig. 10A, B). RvD2 treatment increased GPR18 and MBP levels, while decreasing APP levels (Fig. 10A, B). These results suggest that RvD2 can reduce $\mathrm{SAH}$-induced axonal and myelin injury.

\section{Discussion}

The current study provided a novel angle to investigate the effects of GPR18 on different brain regions at $24 \mathrm{~h}$ after SAH in a rat model. The main findings were as follows: (1) GPR18 expressed in meninges, hypothalamus, cortex and white matter in the brain; (2) SAH increased GPR18 expression in meninges and hypothalamus, but decreased it in cortex and white matter; (3) GPR18 agonist RvD2 mitigated neurological deficits and brain edema induced by SAH; and (4) RvD2 may mediate its neuroprotective effects by activating GPR18 as well as attenuating mast cell degranulation, hypothalamic inflammation, apoptosis, oxidative stress, BBB disruption and WMI in the brain.

SAH has been described as a major cause of poor outcomes in stroke (Orru et al. 2013). Around $85 \%$ of SAH incidents are caused by aneurysm rupture, but the reason for the poor prognosis in SAH remains unclear (Orru et al. 2013). It may reflect EBI during the crucial time window of $72 \mathrm{~h}$ after SAH onset (Rass and Helbok 2019). EBI appears to involve various types of cells in the brain (Chen et al. 2015), so drugs may be more effective against EBI if they can target multiple molecular pathways in different brain regions.

One molecular target may be GPR18, which is expressed by various cell types in different brain regions. It has been implicated in controlling immune system activity and inflammation (Guerrero-Alba et al. 2018); in fact, it may be a useful target in the treatment of inflammatory diseases and cancer (Finlay et al. 2016). GPR18 is expressed in neurons and microvascular endothelial cells in the brain, where it can mitigate injury induced by cerebral ischemia/reperfusion (Zuo et al. 2018). Since GPR18 appears to be expressed by cells in the hypothalamus, it may help protect against SAH in part by targeting mast cell and hypothalamic neurons (Pascoal et al. 2017). Further, previous study also proved that GPR18 functioned through Akt/GSK-3 $\beta$ signal pathway with TNF-a reduction in neurons (Zhang et al. 2018a). Here, we found that GPR18 could improve neurobehavior and brain edema in brain cortex after SAH. We also found that GPR18 is expressed in white matter, which may also be related to its neuroprotective role in white matter injury (WMI) after SAH. On the other hand, our results also prove the anti-inflammatory effects of GPR18, but we did not find evidence that GPR18 is expressed by microglia (Supplemental Figure 2C), consistent with previous work (Pascoal et al. 2017). Thus, the anti-inflammatory and neuroprotective effects of GPR18 may not affect microglial hyperplasia but mast cell degranulation and hypothalamic inflammation.

RvD2 is a novel ligand of GPR18 that can protect second organ lung injury (Chiang et al. 2015). It is a precursor of DHA, which can protect the brain from injury not only by reducing inflammation (Siddiqui et 
al. 2019; Dong et al. 2019; Zuo et al. 2018; Zhang et al. 2018a) but also by exerting other effects (Yin et al. 2016). Indeed, our previous work suggested that DHA could exert anti-oxidant and anti-apoptotic effects soon after SAH (Zhang et al. 2018b), so we wondered whether its precursor RvD2 might exert similar effects to mitigate EBI. Therefore, we tested the effects of RvD2 on SAH-induced BBB disruption, oxidative stress, apoptosis, inflammation and WMI, and found that RvD2 could protect SAH rat brain from these injuries. RvD2 facilitates the metabolism of DHA in the liver, which may help it cross the BBB (Tulowiecka et al. 2020). Thus, using RvD2 in this study would be a better choice to find a rapidly protective effect in the brain after $\mathrm{SAH}$.

While previous research on EBI has focused on cortex, other brain regions are likely involved, given that poor outcomes after SAH have been linked to BBB disruption, oxidative stress, apoptosis, inflammation and WMI (Rass and Helbok 2019; Khey et al. 2020; Pang et al. 2019). Oxidative stress, for example, leads to covalent modification of multiple molecular effectors, altering signal transduction, cytoskeletal organization, and cell adhesion (Cai et al. 2017). Oxidative stress also contributes to endothelial dysfunction, brain edema, neuronal apoptosis as well as acute and chronic inflammation (Zhang et al. 2019a; Echigo et al. 2012). The inflammatory response during acute stress may contribute to the breakdown of the BBB (Leitner 2010). The cerebrospinal fluid of SAH patients shows increased TNF levels in platelets and microvessel endothelial cells, suggesting that cerebral vasospasm triggers inflammatory processes that may contribute to brain injury (Eisenhut 2014). SAH-induced neuronal apoptosis can also be alleviated by protecting the BBB (Liu et al. 2013) and by delivering anti-TNF-a antibody to the hypothalamus (Ma et al. 2018). And hypothalamic inflammation after stroke has been linked to the activity of neuronal POMC and NPY (Pascoal et al. 2017). Mast cell degranulation is part of the early inflammatory response to ischemic and hemorrhage stroke (Strbian et al. 2009; Parrella et al. 2019), and it is a key mediator of acute injury in meninges (Arac et al. 2019).

In our study, the GPR18 ligand RvD2 protected BBB integrity but alleviated oxidative stress and apoptosis, and these effects were associated with up-regulation of GPR18 in cortex. At the same time, RvD2 attenuated WMI, which may be due to the ligand's ability to protect neurofilaments and oligodendrocytes. Our results are consistent with the idea that the anti-inflammatory effects of RvD2 via GPR18 involve hypothalamic neurons and mast cells, but not microglia. We found that RvD2 inhibited mast cell degranulation and hypothalamic inflammation, which in turn reduced TNF-a and elevated IL-10.

There are some limitations of our study. The pathophysiology of EBI is complicated, and the RvD2/GPR18 axis likely involves other proteins related to signaling pathways in mitochondria and other organelles. It is also possible that RvD2 exerts protective effects after SAH through additional brain regions than the four on which we focused here as we did not involve cerebellum and brain stem in sample selection.

Despite these limitations, we present evidence that RvD2 can protect against EBI in a rat model of SAH through activation of GPR18 in multiple brain regions. RvD2 appears to mitigate injury by alleviating 
mast cell degranulation, inflammation, BBB disruption, apoptosis, oxidative stress and WMI. Our study suggests that RvD2 may be an effective treatment for SAH.

\section{Declarations}

\section{Data availability}

All data are fully available without restriction. All relevant data can be found in the paper.

\section{Animal research (ethics)}

All animal procedures were conducted in accordance with the ethical standards of our institution and with national regulations.

\section{Consent to participate (ethics)}

There were no human subjects in this article, so informed consent is not applicable.

\section{Consent to publish (ethics)}

There were no human subjects in this article, so informed consent is not applicable.

\section{Plant reproducibility}

There were no plant subjects in this article, so reproducibility is not applicable.

\section{Clinical trials registration}

There were no human subjects in this article, so trial registration is not applicable.

\section{Author contributions}

Tongyu Zhang designed the study, performed the experiments and wrote the manuscript. Gang Zuo contributed to data analysis. Hongqi Zhang contributed to research design. All authors analyzed the results and approved the final version of the manuscript.

\section{Conflicts of interest}

The authors declare no potential conflicts of interest with respect to the research, authorship, or publication of this article.

\section{Funding}

This research was supported by grants from the China Postdoctoral Science Foundation to TY Zhang (2019TQ0216 and 2019M660716), the Beijing Postdoctoral Science Foundation to TY Zhang (2020-ZZ020), the National Natural Science Foundation of China to Dr. TY Zhang (82001324). And the Jiangsu 
Provincial Health and Family Planning Commission of China to G Zuo (QNRC2016263, H201654, and GSWS2019080).

\section{References}

1. Arac A, Grimbaldeston MA, Galli SJ, Bliss TM, Steinberg GK (2019), Meningeal Mast Cells as Key Effectors of Stroke Pathology. Front Cell Neurosci 13:126.

2. Cai J, Xu D, Bai X, Pan R, Wang B, Sun S, Chen R, Sun J, et al. (2017), Curcumin mitigates cerebral vasospasm and early brain injury following subarachnoid hemorrhage via inhibiting cerebral inflammation. Brain Behav 7:e00790.

3. Chen S, Feng H, Sherchan P, Klebe D, Zhao G, Sun X, Zhang J, Tang J, et al. (2014), Controversies and evolving new mechanisms in subarachnoid hemorrhage. Prog Neurobiol 115:64-91.

4. Chen S, Luo J, Reis C, Manaenko A, Zhang J (2017), Hydrocephalus after Subarachnoid Hemorrhage: Pathophysiology, Diagnosis, and Treatment. Biomed Res Int 2017:8584753.

5. Chen S, Wu H, Tang J, Zhang J, Zhang JH (2015), Neurovascular events after subarachnoid hemorrhage: focusing on subcellular organelles. Acta Neurochir Suppl 120:39-46.

6. Chiang N, Dalli J, Colas RA, Serhan CN (2015), Identification of resolvin D2 receptor mediating resolution of infections and organ protection. J Exp Med 212:1203-1217.

7. Ciurea, A.V., Palade, C., Voinescu, D., and Nica, D.A. (2013). Subarachnoid hemorrhage and cerebral vasospasm - literature review. J Med Life 6, 120-125.

8. Dong X, Gao J, Zhang CY, Hayworth C, Frank M, Wang Z (2019), Neutrophil Membrane-Derived Nanovesicles Alleviate Inflammation To Protect Mouse Brain Injury from Ischemic Stroke. ACS nano 13:1272-1283.

9. Dunn SL, Wilkinson JM, Crawford A, Bunning RAD, Le Maitre CL (2016), Expression of Cannabinoid Receptors in Human Osteoarthritic Cartilage: Implications for Future Therapies. Cannabis Cannabinoid Res 1:3-15.

10. Echigo R, Shimohata N, Karatsu K, Yano F, Kayasuga-Kariya Y, Fujisawa A, Ohto T, Kita Y, et al. (2012), Trehalose treatment suppresses inflammation, oxidative stress, and vasospasm induced by experimental subarachnoid hemorrhage. J Transl Med 10:80.

11. Eisenhut M. (2014). Vasospasm in cerebral inflammation. Int J Inflam 509707.

12. Finlay DB, Joseph WR, Grimsey NL, Glass M (2016), GPR18 undergoes a high degree of constitutive trafficking but is unresponsive to N-Arachidonoyl Glycine. PeerJ 4:e1835.

13. Guerrero-Alba R, Barragan-Iglesias P, Gonzalez-Hernandez A, Valdez-Morales EE, Granados-Soto V, Condes-Lara M, Rodriguez MG, Marichal-Cancino BA (2018), Some Prospective Alternatives for Treating Pain: The Endocannabinoid System and Its Putative Receptors GPR18 and GPR55. Front Pharmacol 9:1496.

14. Khey KMW, Huard A, Mahmoud SH (2020), Inflammatory Pathways Following Subarachnoid Hemorrhage. Cell Mol Neurobiol 40:675-693. 
15. Leitner H (2010), Influence of neurosteroids on the pathogenesis of multiple sclerosis. Med Hypotheses 75:229-234.

16. Liu Y, Cai H, Wang Z, Li J, Wang K, Yu Z, Chen G (2013), Induction of autophagy by cystatin C: a potential mechanism for prevention of cerebral vasospasm after experimental subarachnoid hemorrhage. Eur J Med Res 18:21.

17. Ma L, Jiang Y, Dong Y, Gao J, Du B, Liu D (2018), Anti-TNF-alpha antibody attenuates subarachnoid hemorrhage-induced apoptosis in the hypothalamus by inhibiting the activation of Erk. Neuropsychiatr Dis Treat 14:525-536.

18. Macdonald RL, Schweizer TA (2017), Spontaneous subarachnoid haemorrhage. Lancet 389:655-666.

19. Orru E, Roccatagliata L, Cester G, Causin F, Castellan L (2013), Complications of endovascular treatment of cerebral aneurysms. Eur J Radiol 82:1653-1658.

20. Pang J, Peng J, Matei N, Yang P, Kuai L, Wu Y, Chen L, Vitek MP, et al. (2018), Apolipoprotein E Exerts a Whole-Brain Protective Property by Promoting M1? Microglia Quiescence After Experimental Subarachnoid Hemorrhage in Mice. Translational stroke research 9:654-668.

21. Pang J, Peng J, Yang P, Kuai L, Chen L, Zhang JH, Jiang Y (2019), White Matter Injury in Early Brain Injury after Subarachnoid Hemorrhage. Cell Transplant 28:26-35.

22. Parrella E, Porrini V, Benarese M, Pizzi M (2019), The Role of Mast Cells in Stroke. Cells 8.

23. Pascoal LB, Bombassaro B, Ramalho AF, Coope A, Moura RF, Correa-da-Silva F, Ignacio-Souza L, Razolli D, et al. (2017), Resolvin RvD2 reduces hypothalamic inflammation and rescues mice from diet-induced obesity. J Neuroinflammation 14:5.

24. Rass V, Helbok R (2019), Early Brain Injury After Poor-Grade Subarachnoid Hemorrhage. Curr Neurol Neurosci Rep 19:78.

25. Siddiqui YD, Omori K, Ito T, Yamashiro K, Nakamura S, Okamoto K, Ono M, Yamamoto T, et al. (2019), Resolvin D2 Induces Resolution of Periapical Inflammation and Promotes Healing of Periapical Lesions in Rat Periapical Periodontitis. Front Immunol 10:307.

26. Strbian D, Kovanen PT, Karjalainen-Lindsberg ML, Tatlisumak T, Lindsberg PJ (2009), An emerging role of mast cells in cerebral ischemia and hemorrhage. Ann Med 41:438-450.

27. Topkoru B, Egemen E, Solaroglu I, Zhang JH (2017), Early Brain Injury or Vasospasm? An Overview of Common Mechanisms. Curr Drug Targets 18:1424-1429.

28. Tulowiecka N, Kotlega D, Prowans P, Szczuko M (2020), The Role of Resolvins: EPA and DHA Derivatives Can Be Useful in the Prevention and Treatment of Ischemic Stroke. Int J Mol Sci 21.

29. Wilhelmsen K, Khakpour S, Tran A, Sheehan K, Schumacher M, Xu F, Hellman J (2014), The endocannabinoid/endovanilloid N-arachidonoyl dopamine (NADA) and synthetic cannabinoid WIN55,212-2 abate the inflammatory activation of human endothelial cells. J Biol Chem 289:1307913100.

30. Yehya M, Torbey MT (2018), The Role of Mast Cells in Intracerebral Hemorrhage. Neurocrit Care 28:288-295. 
31. Yin J, Li H, Meng C, Chen D, Chen Z, Wang Y, Wang Z, Chen G (2016), Inhibitory effects of omega-3 fatty acids on early brain injury after subarachnoid hemorrhage in rats: Possible involvement of $G$ protein-coupled receptor 120/beta-arrestin2/TGF-beta activated kinase-1 binding protein-1 signaling pathway. Int J Biochem Cell Biol 75:11-22.

32. Zhang LY, Liu ZH, Zhu Q, Wen S, Yang CX, Fu ZJ, Sun T (2018), Resolvin D2 Relieving Radicular Pain is Associated with Regulation of Inflammatory Mediators, Akt/GSK-3beta Signal Pathway and GPR18. Neurochem Res 43:2384-2392.

33. Zhang T, Wu P, Budbazar E, Zhu Q, Sun C, Mo J, Peng J, Gospodarev V, et al. (2019), Mitophagy Reduces Oxidative Stress Via Keap1 (Kelch-Like Epichlorohydrin-Associated Protein 1)/Nrf2 (Nuclear Factor-E2-Related Factor 2)/PHB2 (Prohibitin 2) Pathway After Subarachnoid Hemorrhage in Rats. Stroke 50:978-988.

34. Zhang T, Wu P, Zhang JH, Li Y, Xu S, Wang C, Wang L, Zhang G, et al. (2018), Docosahexaenoic Acid Alleviates Oxidative Stress-Based Apoptosis Via Improving Mitochondrial Dynamics in Early Brain Injury After Subarachnoid Hemorrhage. Cell Mol Neurobiol 38:1413-1423.

35. Zhang T, Xu S, Wu P, Zhou K, Wu L, Xie Z, Xu W, Luo X, et al. (2019), Mitoquinone attenuates bloodbrain barrier disruption through Nrf2/PHB2/OPA1 pathway after subarachnoid hemorrhage in rats. Exp Neurol 317:1-9.

36. Zhang YL, Zhang PB, Qiu SD, Liu Y, Tian YF, Wang Y (2006), Effects of ketamine-midazolam anesthesia on the expression of NMDA and AMPA receptor subunit in the peri-infarction of rat brain. Chin Med J (Engl) 119:1555-1562.

37. Zuo G, Zhang D, Mu R, Shen H, Li X, Wang Z, Li H, Chen G (2018), Resolvin D2 protects against cerebral ischemia/reperfusion injury in rats. Mol Brain 11:9.

\section{Figures}




\section{Experiment 1}
(1) Naïve
$(n=3)$
(2) Sham
$(n=9)$
(3) $\mathrm{SAH}-3 \mathrm{~h}$
$(n=6)$
IF
(4) $\mathrm{SAH}-6 \mathrm{~h}$
$(n=6)$
(5) $\mathrm{SAH}-12 \mathrm{~h}$
$(n=6)$
(6) $\mathrm{SAH}-24 \mathrm{~h}$
$(n=9)$
(7) $\mathrm{SAH}-72 \mathrm{~h}$
$(n=6)$
WB
(meninges/hypothalamus/cortex/white matter)

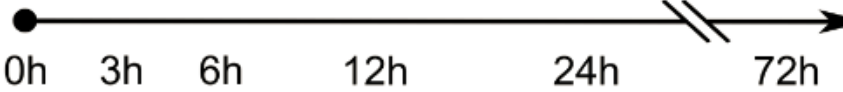

\section{Experiment 2}
(1) Sham+vehicle
(2) $\mathrm{SAH}+$ vehicle
(3) $\mathrm{SAH}+\mathrm{RvD2}(0.3 \mu \mathrm{g} / \mathrm{kg})$

$$
\begin{aligned}
& (n=6) \\
& (n=6) \\
& (n=6) \\
& (n=6) \\
& (n=6)
\end{aligned}
$$
(4) $\mathrm{SAH}+\mathrm{RvD} 2(0.9 \mu \mathrm{g} / \mathrm{kg})$
(5) $\mathrm{SAH}+\mathrm{RvD2}(2.7 \mu \mathrm{g} / \mathrm{kg})$

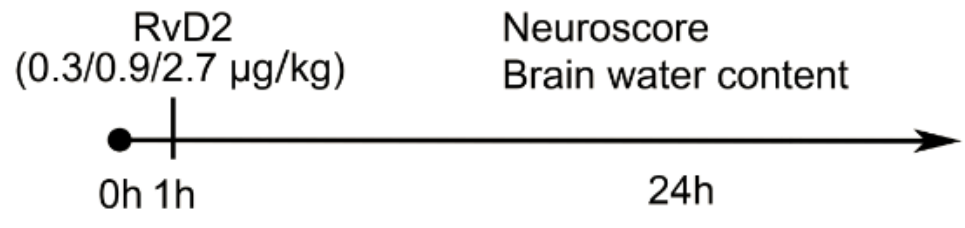

\section{Experiment 3}

(1) Sham+vehicle

$(n=12)$

\begin{tabular}{|c|c|}
\hline $\begin{array}{c}\text { RvD2 } \\
\text { (Best dosage) }\end{array}$ & $\begin{array}{l}\text { Neuroscore } \\
\text { IF,IHC,TUNEL,DHE } \\
\text { WB }\end{array}$ \\
\hline Oh $1 \mathrm{~h}$ & $24 \mathrm{~h}$ \\
\hline
\end{tabular}

(2) $\mathrm{SAH}+$ vehicle

$(n=12)$

(3) $\mathrm{SAH}+\mathrm{RvD2}$

$(n=12)$

Figure 1

Experimental design of the entire study. IF, Immunofluorescence; WB, western blot; TUNEL, terminal deoxynucleotidyl transferase dUTP; IHC, immunohistochemistry; DHE, dihydroethidium. 
a

\section{Groups}

Experiment 1
(1) Naïve
(2) Sham
(3) $\mathrm{SAH}-3 \mathrm{~h}$
(4) $\mathrm{SAH}-6 \mathrm{~h}$
(5) $\mathrm{SAH}-12 \mathrm{~h}$
(6) $\mathrm{SAH}-24 \mathrm{~h}$
(7) $\mathrm{SAH}-72 \mathrm{~h}$

\section{Experiment 2}

(1) Sham+vehicle

(2) $\mathrm{SAH}+$ vehicle

(3) $\mathrm{SAH}+\mathrm{RvD} 2(0.3 \mu \mathrm{g} / \mathrm{kg})$

(4) $\mathrm{SAH}+\mathrm{RvD2}(0.9 \mu \mathrm{g} / \mathrm{kg})$

(5) $\mathrm{SAH}+\mathrm{RvD} 2(2.7 \mu \mathrm{g} / \mathrm{kg})$

\section{Experiment 3}

(1) Sham+vehicle

(2) $\mathrm{SAH}+$ vehicle

(3) $\mathrm{SAH}+\mathrm{RvD2}$

Total

Naïve, Sham

$\mathrm{SAH}, \mathrm{SAH}+$ vehicle

$\mathrm{SAH}+\mathrm{RvD2}$

\section{Mortality Rate Excluded}

$0(0 / 3)$

$0(0 / 9)$

$\begin{array}{cc}22.22 \%(2 / 9) & 1 \\ 27.27 \%(3 / 11) & 2 \\ 25 \%(2 / 8) & \\ 28.57 \%(4 / 14) & 1 \\ 14.29 \%(1 / 7) & \end{array}$

1

b

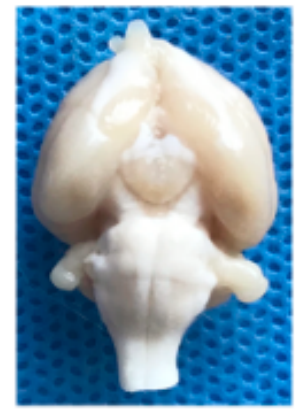

Sham

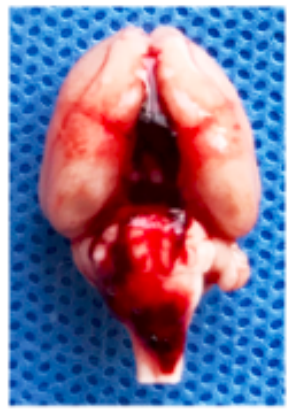

SAH

\section{C}

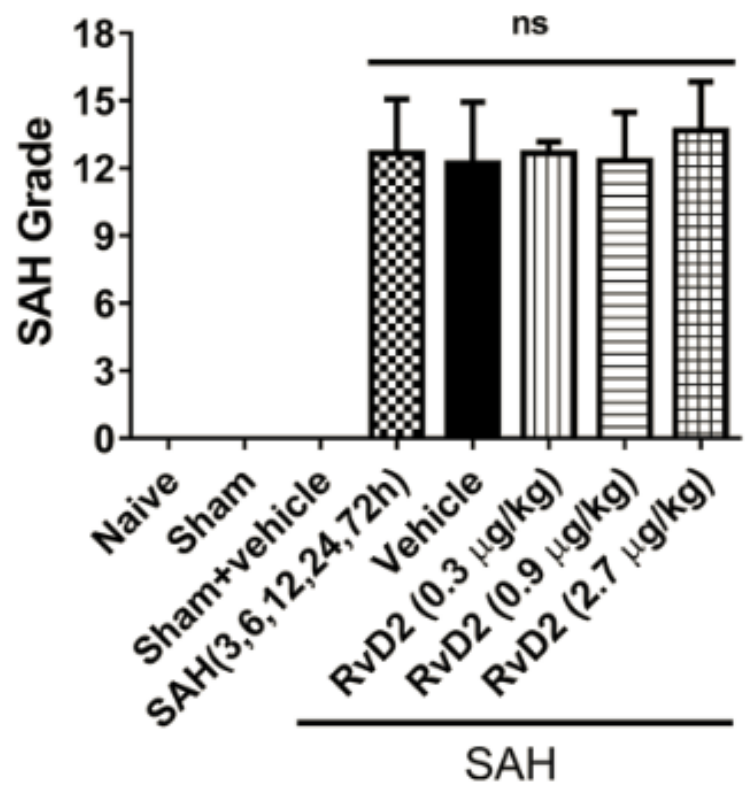

Figure 2

. (A) Mortality rates in the different groups in each experiment. "Excluded" refers to animals excluded from analysis because low SAH grade. (B) Photographs of representative whole brains from Sham and SAH groups. (C) SAH grades. Bars show mean \pm SD. ns, no statistical significance. 

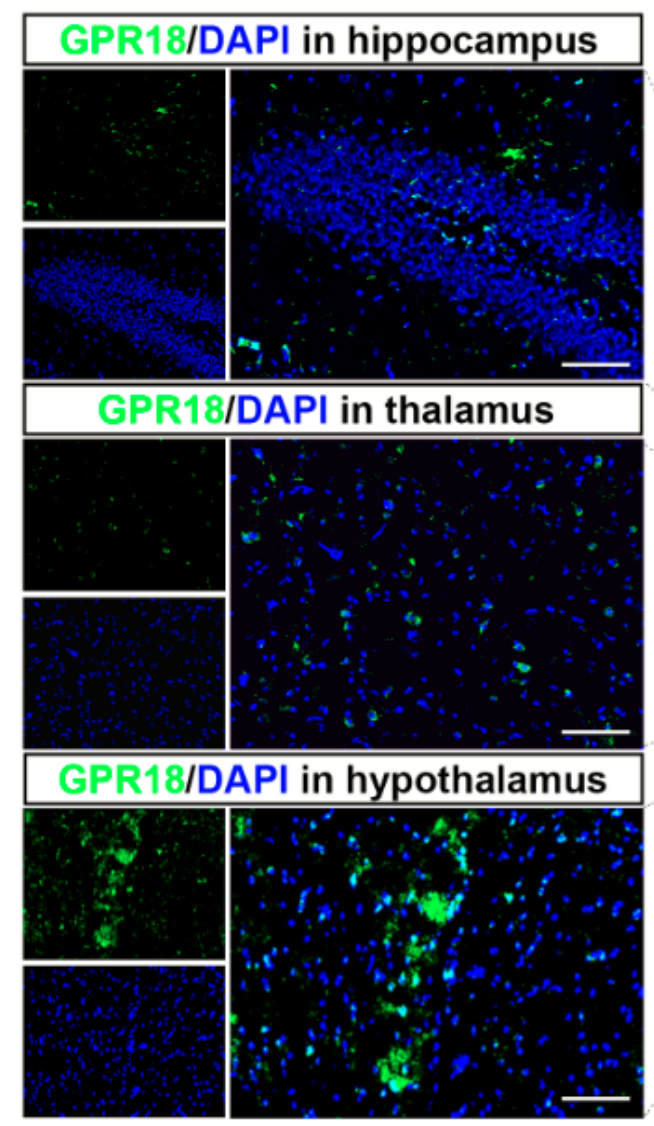
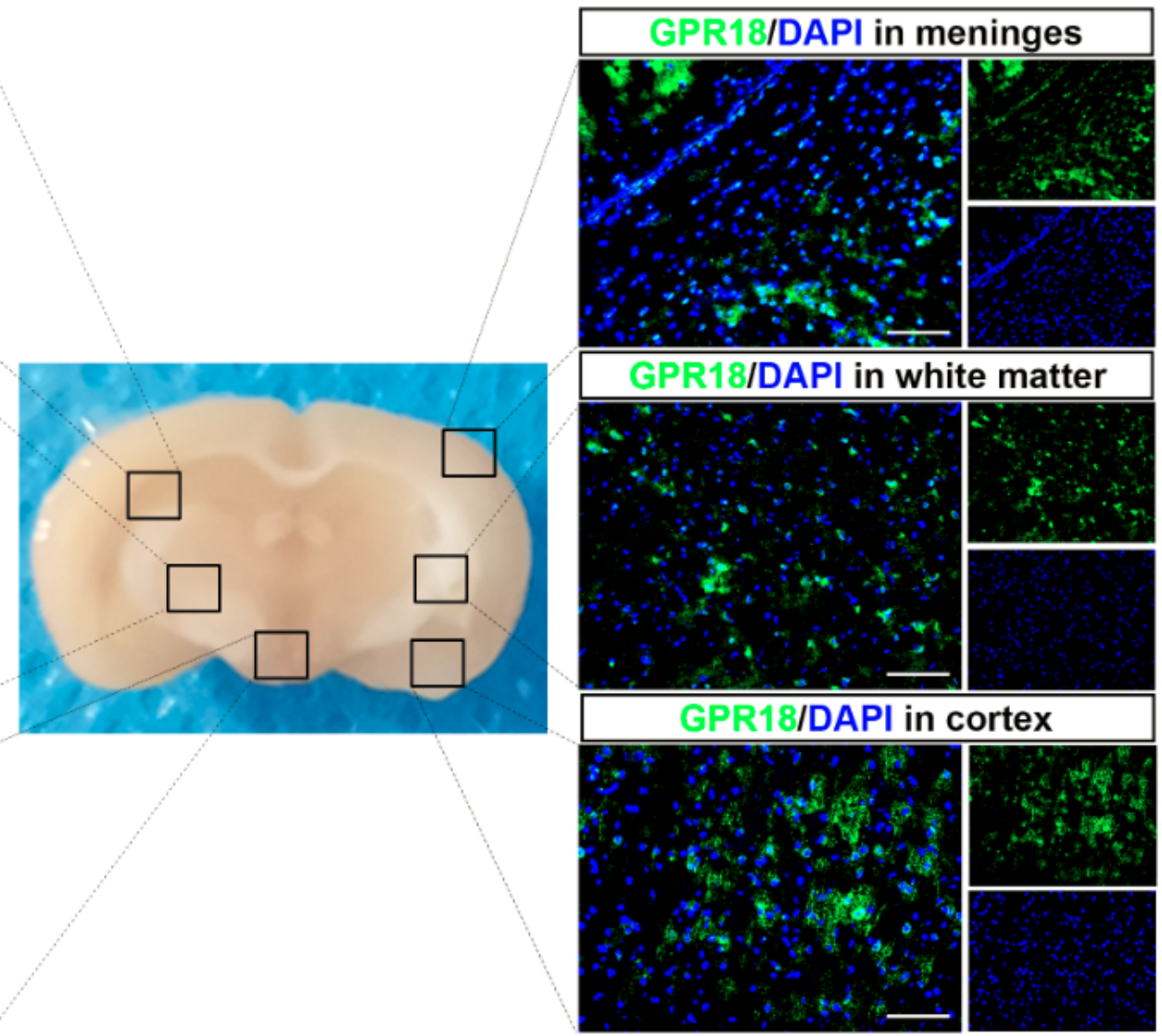

Figure 3

Expression of GPR18 (green) in different brain regions in the Naïve group. Nuclei were counterstained using DAPI (blue). The results shown are representative of those obtained with three animals. Scale bars $=50 \mu \mathrm{m}$.

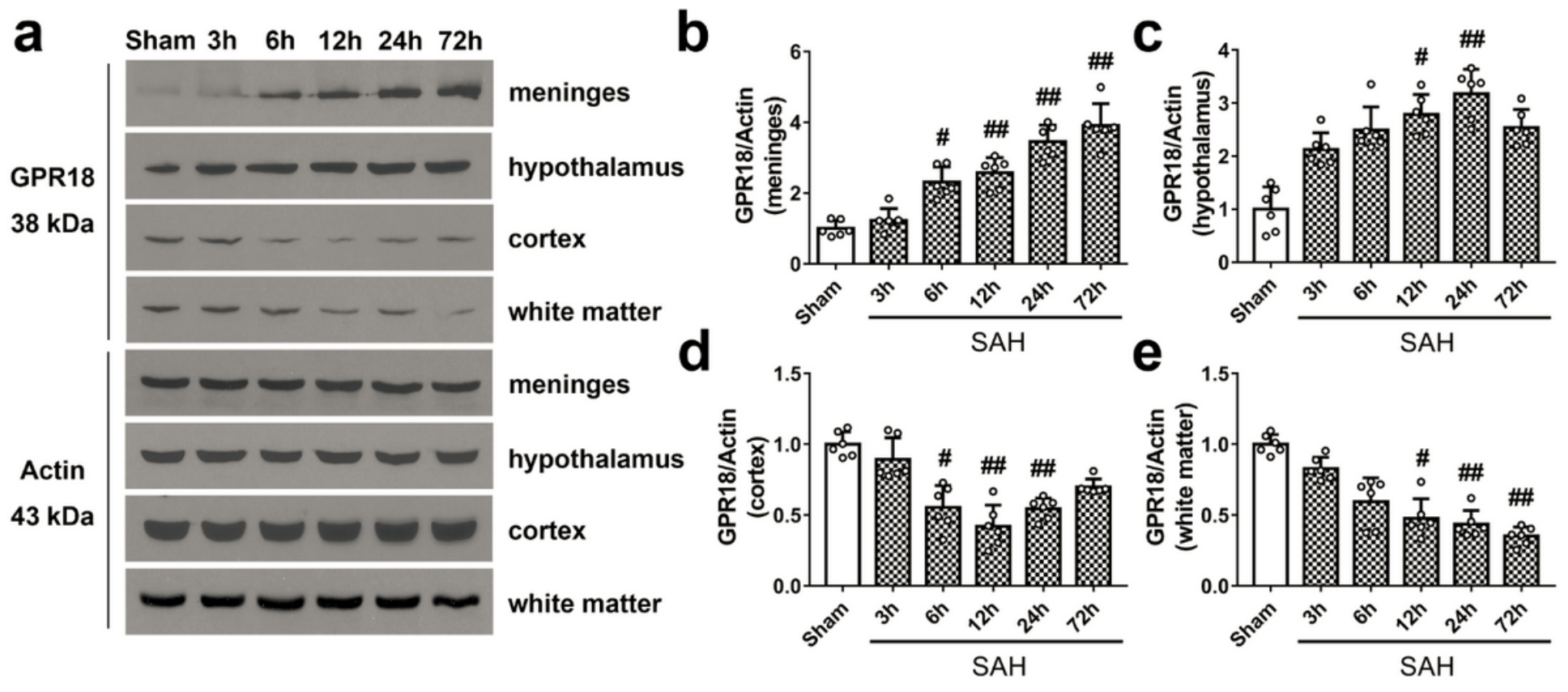

Figure 4 
Time course of GPR18 expression in different brain regions after SAH. (A) Representative Western blot results in the Sham group and SAH groups at 3, 6, 12, 24, and $72 \mathrm{~h}$. (B-E) Quantitative analysis of GPR18 expression in meninges, hypothalamus, cortex and white matter. Bars show mean $\pm S D(n=6$ from each group). \#\# $\mathrm{P}<0.01$, \# $\mathrm{P}<0.05$ vs. Sham group.
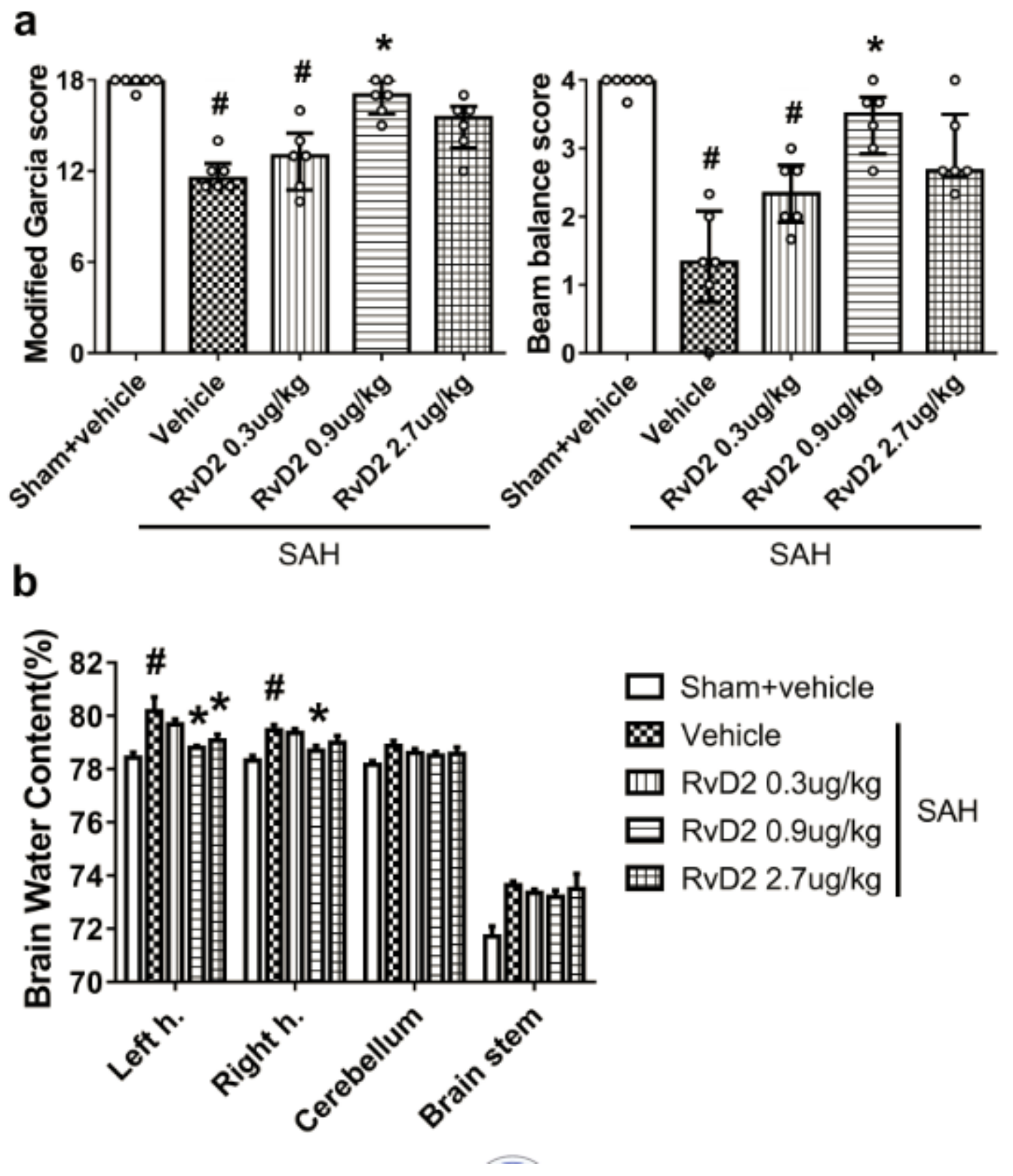

Figure 5

RvD2 improved neurological scores and brain edema after SAH. (A) Modified Garcia and beam balance scores. Bars represent the median \pm interquartile range ( $n=6$ from each group). (B) Brain water content. Bars represent mean $\pm S D\left(n=6\right.$ from each group). \# $P<0.05$ vs. Sham + vehicle group; ${ }^{*} P<0.05$ vs. $\mathrm{SAH}+$ vehicle group. 
a

Toluidine

blue

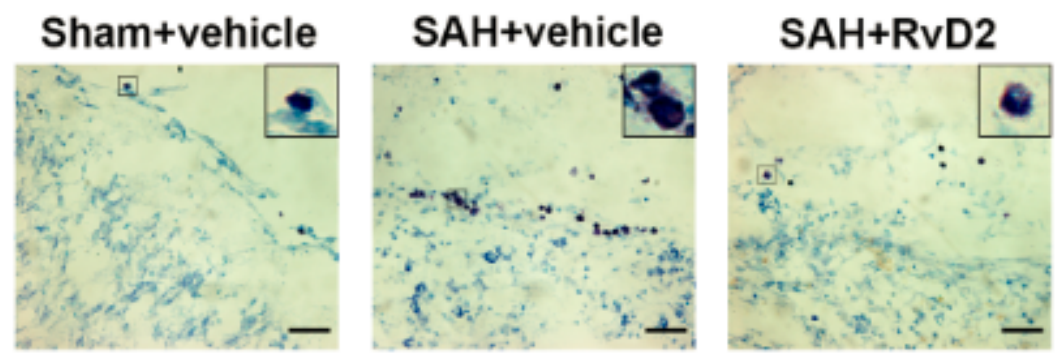

b
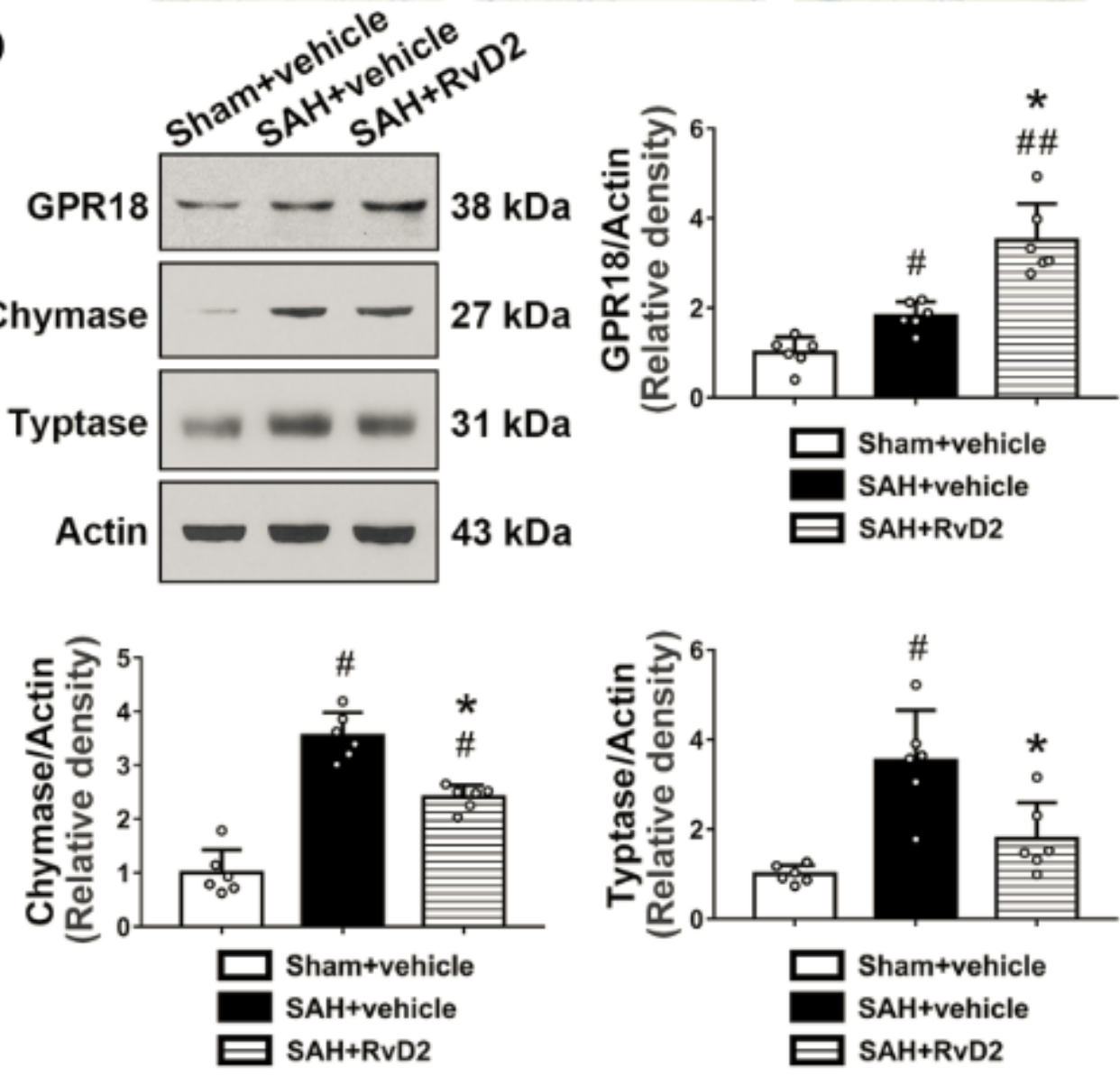

Figure 6

RvD2 attenuated mast cell degranulation in meninges after SAH. (A) Representative meninges sections stained with toluidine blue to reveal mast cell degranulation. Scale bars $=200 \mu \mathrm{m}$. (B) Representative Western blot bands and quantitative analysis of chymase and tryptase expression in meninges. Bars represent mean $\pm S D$ ( $n=6$ for each group). \# $P<0.05$, \#\# $P<0.01$ vs. Sham + vehicle group; * $P<0.05$ vs. $\mathrm{SAH}+$ vehicle group. 
a

Hypothalamus $C_{x}=0$

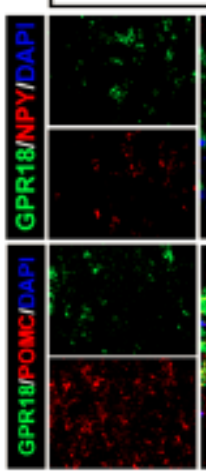
Sham+vehicle
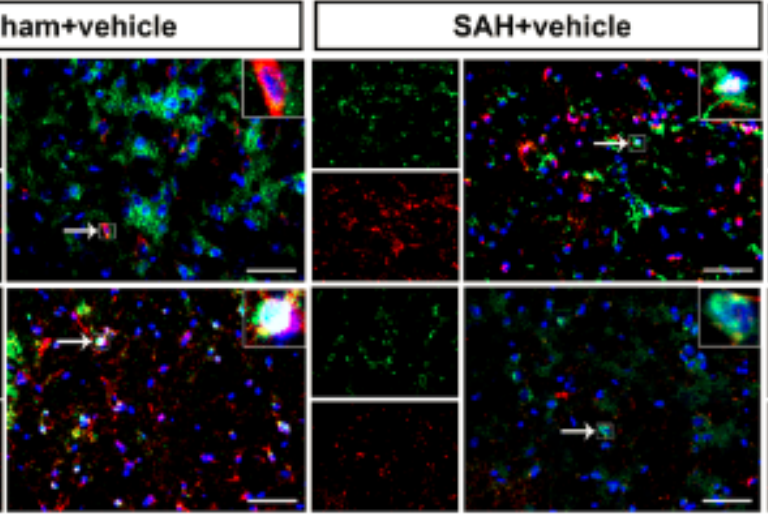

b
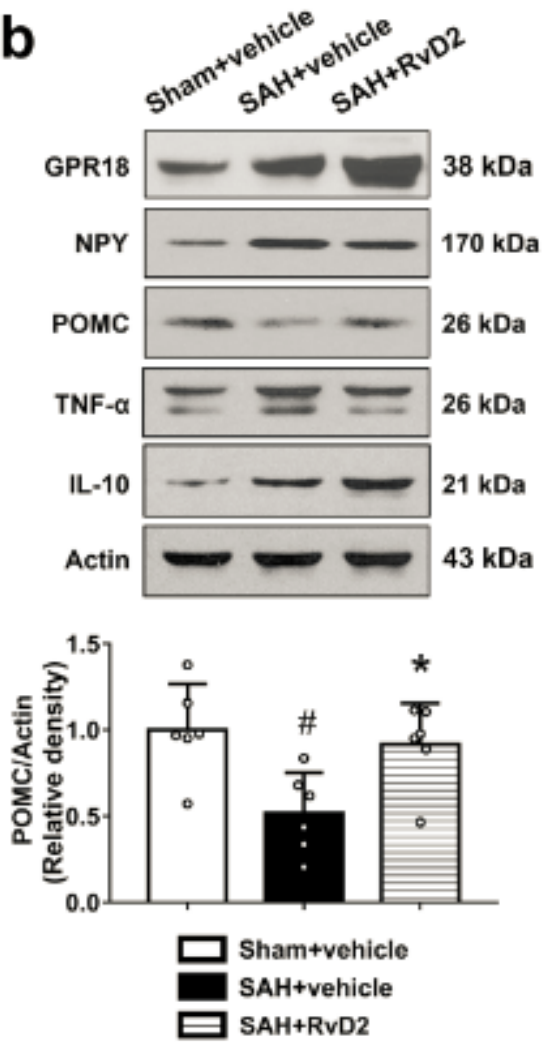
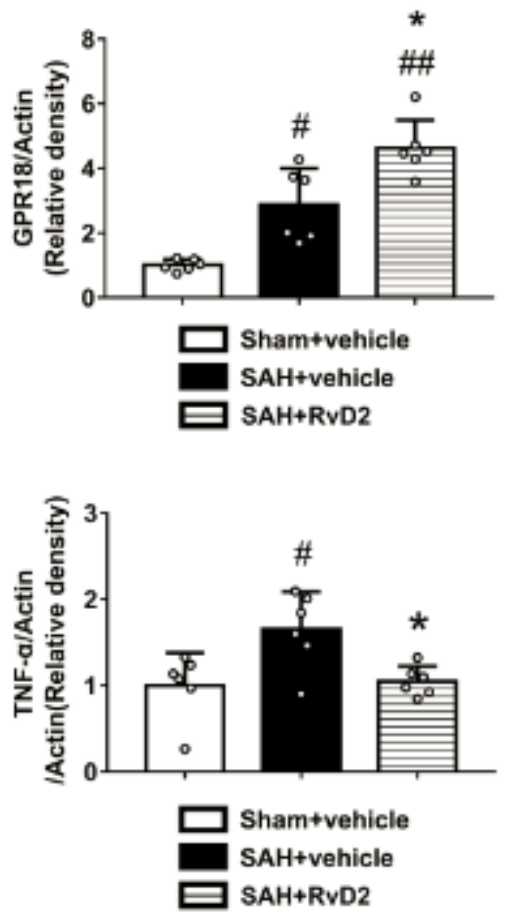
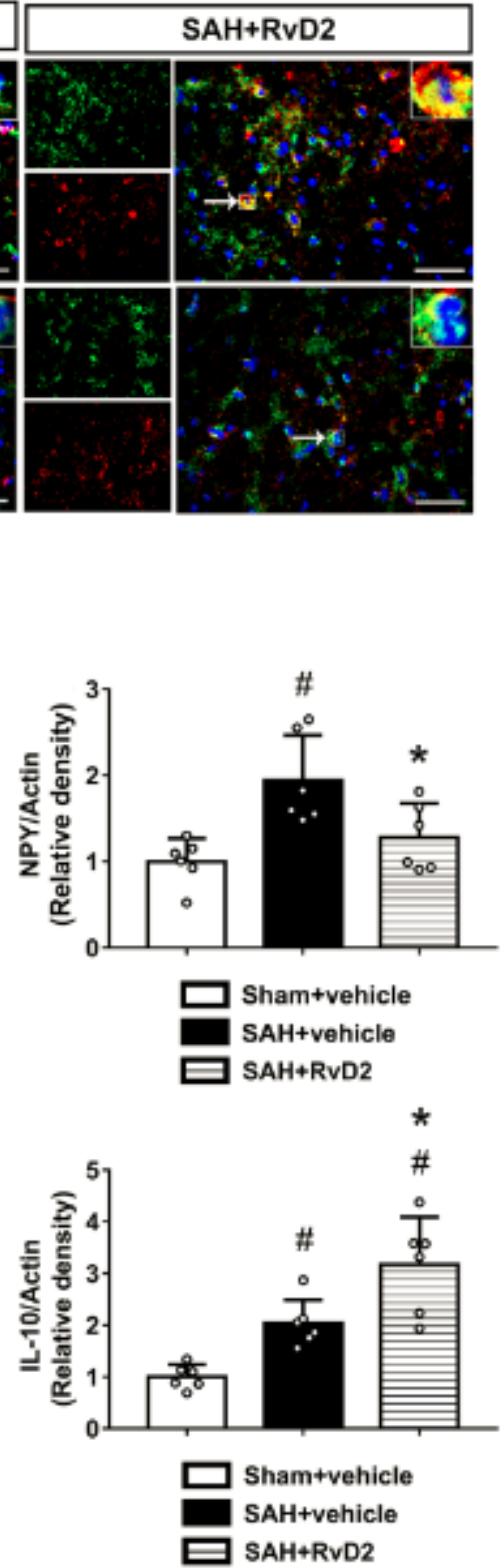

Figure 7

RvD2 attenuated inflammation in hypothalamus after SAH. (A) Double immunofluorescence staining for GPR18 (green) and NPY (red) or POMC (red) in hypothalamus. Scale bars $=50 \mu \mathrm{m}$. (A) Representative Western blot bands and quantitative analysis of GPR18, NPY, POMC, TNF-a, and IL-10 in hypothalamus. Bars represent mean $\pm S D(n=6$ from each group). \# $P<0.05$, \#\# $P<0.01$ vs. Sham + vehicle group; $P<$ 0.05 vs. $\mathrm{SAH}+$ vehicle group. 


\section{a}

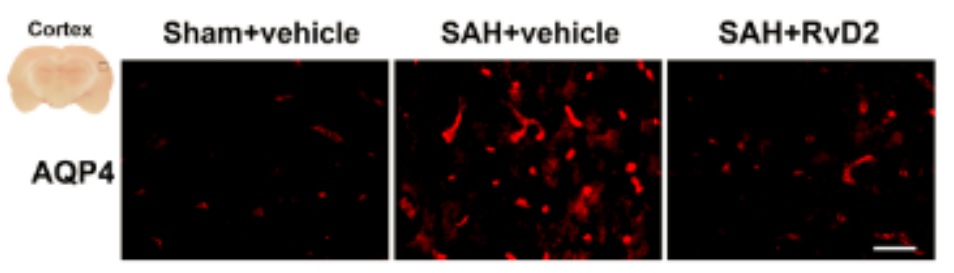

b

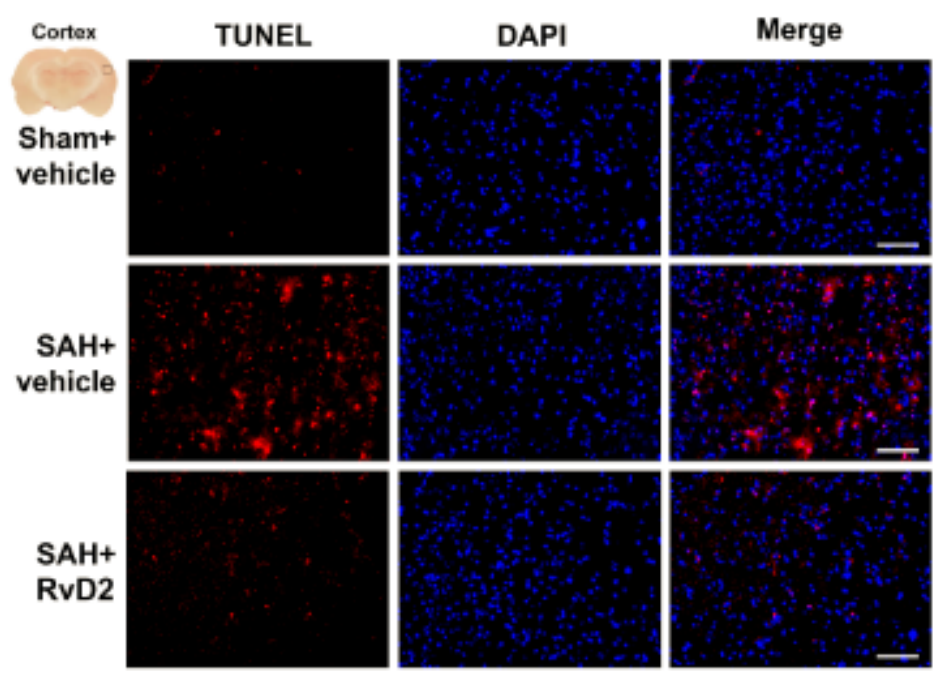

\section{C}

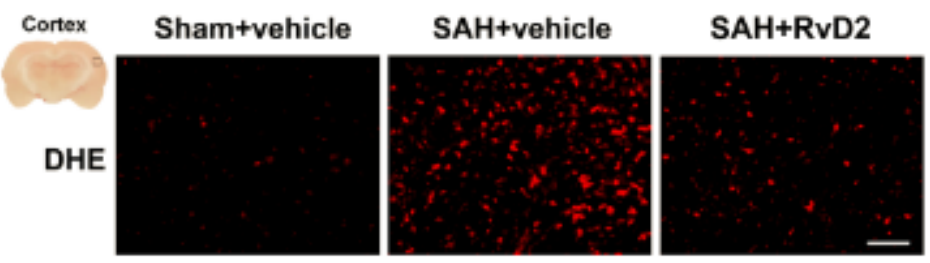

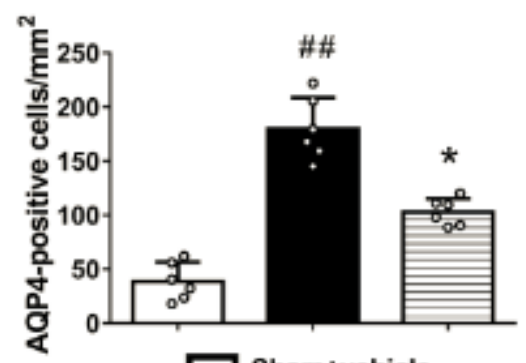

Sham+vehicle

SAH+vehicle

SAH+RvD2
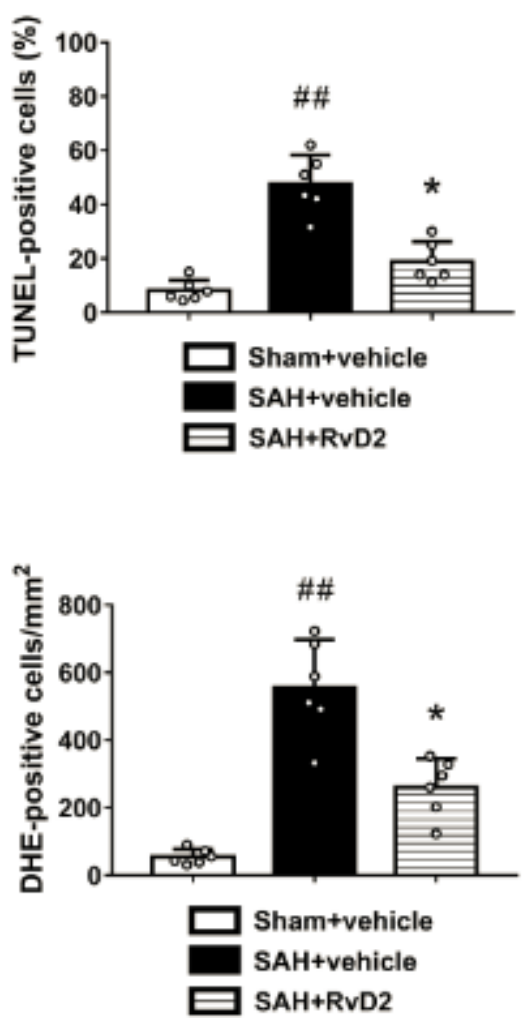

\section{Figure 8}

. Immunostaining studies to show that RvD2 protected BBB and attenuated apoptosis and oxidative stress in cortex after SAH. (A) Representative immunostaining for AQP4, and quantitative analysis of AQP4-positive cells. (B) Representative TUNEL staining, and quantitative analysis of apoptotic index (percentage of TUNEL-positive cells). (C) Representative DHE staining, and quantitative analysis. Scale bars $=200 \mu \mathrm{m}$. Bars represent the mean $\pm S D$ ( $n=6$ from each group). \#\# $P<0.01$ vs. Sham + vehicle group; * $P<0.05$ vs. $S A H+$ vehicle group. 


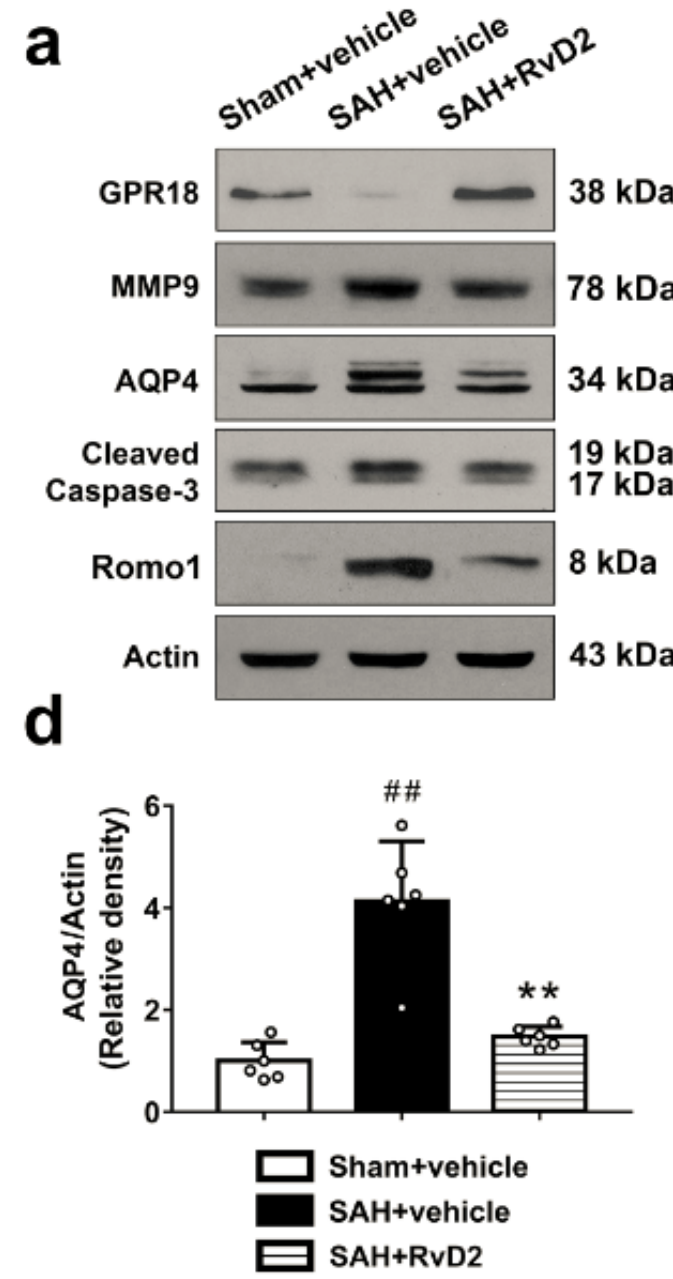

b c
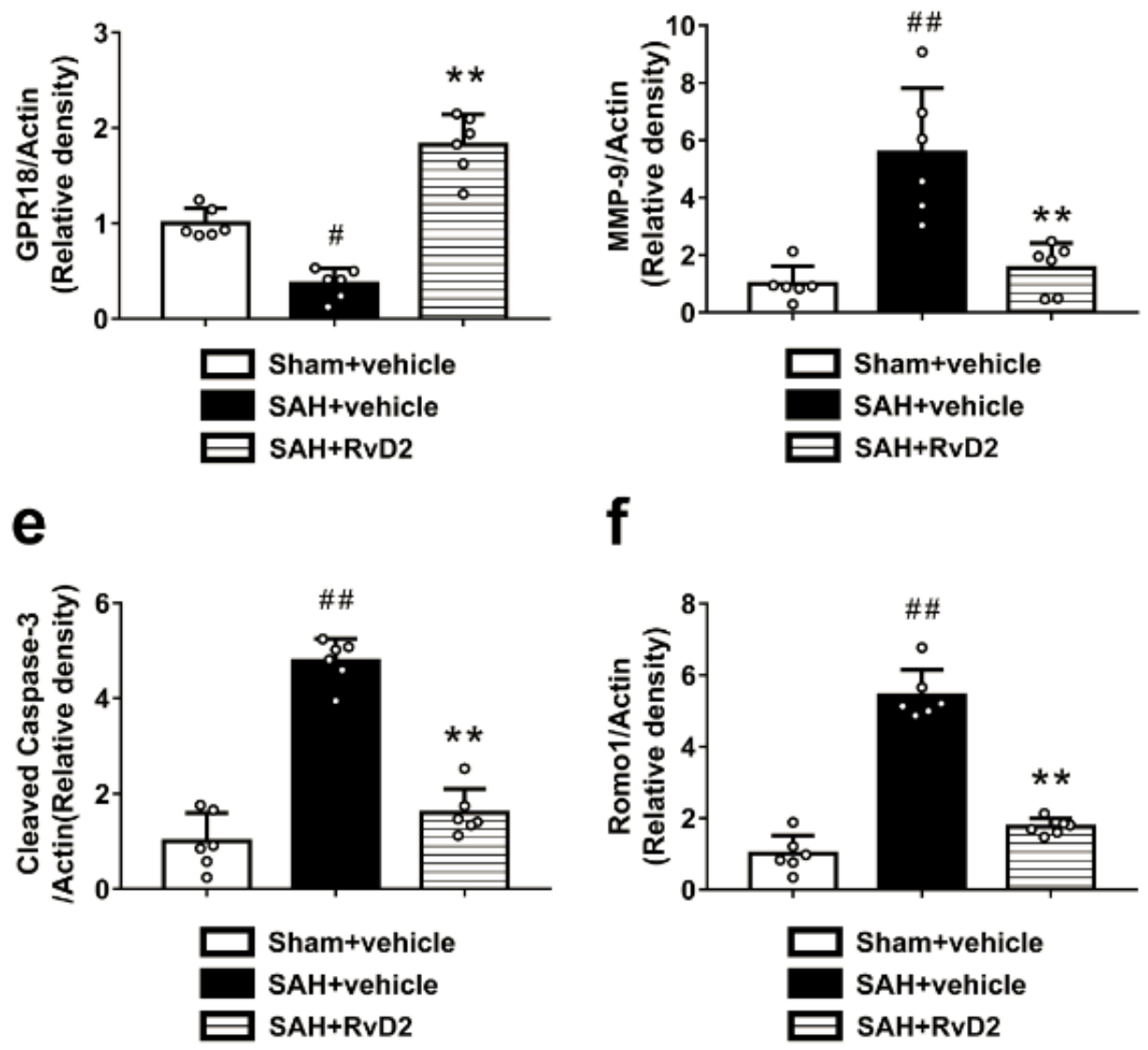

Figure 9

Western blotting studies to show that RvD2 protected BBB as well as attenuated apoptosis and oxidative stress in cortex after SAH. (A) Representative Western blots of GPR18, MMP-9, AQP4, cleaved caspase-3, and Romo1 in cortex. (B-F) Quantitative analysis of the Western blots for each protein. Bars represent mean $\pm S D(n=6$ from each group). \# $P<0.05$, \#\# $P<0.01$ vs. Sham + vehicle group; $* \star P<0.01$ vs. SAH + vehicle group. 


\section{a}
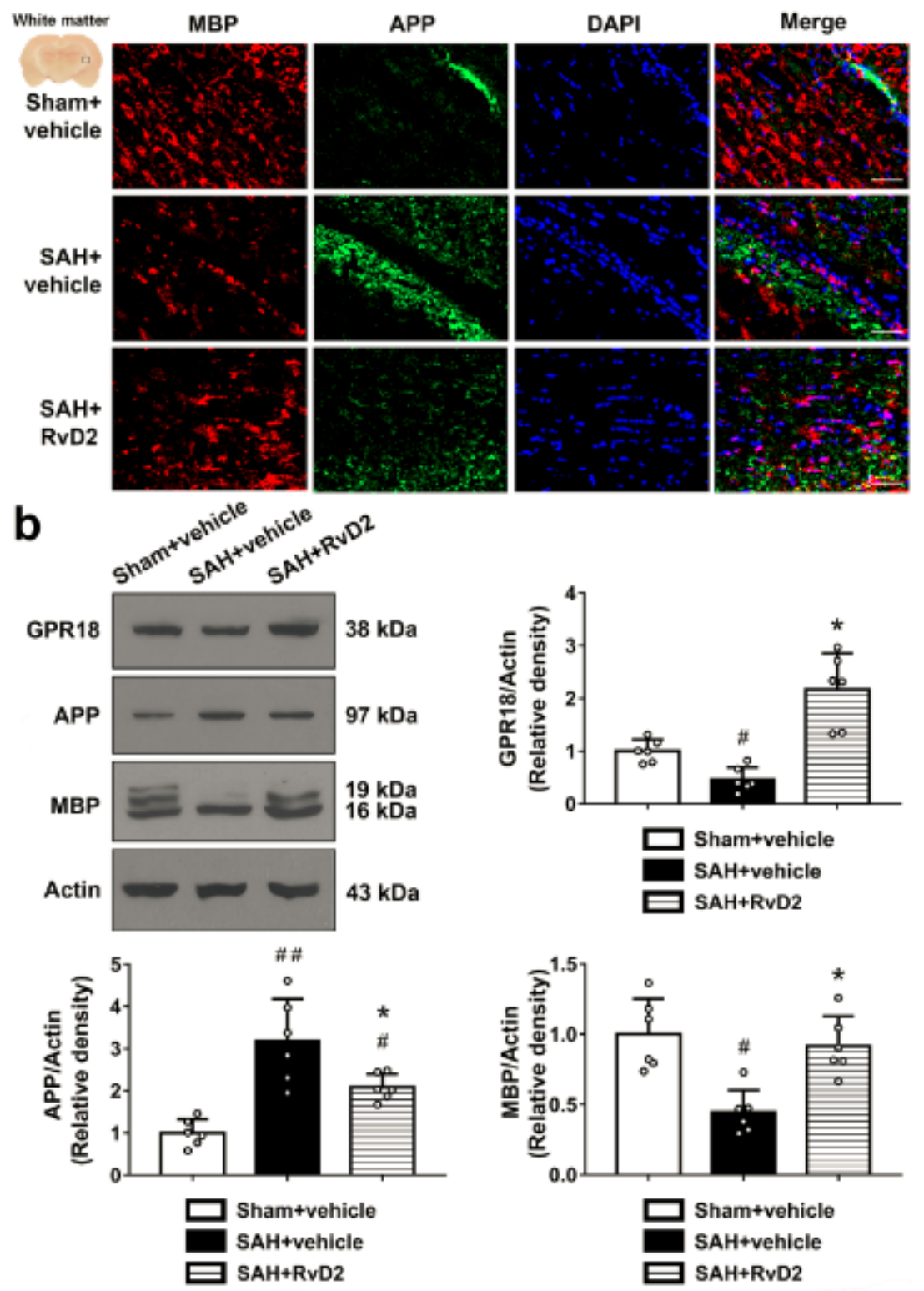

Figure 10

RvD2 mitigated axonal and myelin injury in white matter after SAH. (A) Representative MBP/APP staining to detect white matter injury. (B) Representative Western blot bands and quantitative analysis of GPR18, APP and MBP. Scale bars $=100 \mu \mathrm{m}$. Bars represent mean \pm SD $(n=6$ from each group). \# P<0.05, \#\# P $<0.01$ vs. Sham + vehicle group; ${ }^{*} P<0.05$ vs. $S A H+$ vehicle group.

\section{Supplementary Files}

This is a list of supplementary files associated with this preprint. Click to download.

- figs1.png

- figs2.png 
- figs3.png

Page 24/24 